\title{
The thick disc component of the Galaxy from near infrared colour-magnitude diagrams
}

\author{
A. Cabrera-Lavers ${ }^{1}$, F. Garzón ${ }^{1,2}$, and P. L. Hammersley ${ }^{1}$ \\ 1 Instituto de Astrofísica de Canarias, 38200 La Laguna, Tenerife, Spain \\ e-mail: acabrera@ll.iac.es \\ 2 Departamento de Astrofísica, Universidad de La Laguna, 38200 La Laguna, Tenerife, Spain
}

Received 7 May 2004 / Accepted 8 December 2004

\begin{abstract}
The vertical distribution of $K$ giants stars is analyzed using 2MASS data and a semiempirical method of extracting stellar densities from the $K / J-K$ colour-magnitude diagrams. The vertical density distribution of red clump giant stars can be described by a double exponential, with scaleheights of $268.81 \pm 12.65 \mathrm{pc}$ and $1061.9 \pm 52.16 \mathrm{pc}$. These values are in agreement with the expected values for the thin and thick disc components of the Milky Way. For the thick disc, the radial scalelength is also obtained, with a value of $h_{R}=3.04 \pm 0.11 \mathrm{kpc}$.
\end{abstract}

Key words. Galaxy: general - Galaxy: stellar content - Galaxy: structure - infrared: stars - Galaxy: disk

\section{Introduction}

It is well known that the stellar density in the direction perpendicular to the Galactic plane shows a significative change in the slope at heights of 1-2 kpc above the Plane. Gilmore \& Reid (1983) were the first to suggest that the excess of stars at $1 \mathrm{kpc}$ above the plane corresponds to a different stellar population, which is known as the thick disc. This is probably related to Population II stars, although this claim remains controversial. The change in the luminosity function with the vertical distance above the plane shows that the thick disc is an old population component of the Galaxy, with a metallicity compatible with those of the globular clusters of the disc (Robin et al. 1996; Buser et al. 1999; Vallenari et al. 2000).

Since this new component was defined, the real nature of this population has been widely discussed. Some argue that it is a completely separate population (Gilmore \& Wyse 1985; Gilmore et al. 1989; Carney et al. 1989) whilst others that it corresponds to the stars with the highest velocity dispersions of the old disc (Norris \& Green 1989; Norris \& Ryan 1991). In either case, this "extended disc" with a high value of scale height comprises the oldest stars of the disc, which have suffered the more intense scattering process from the Galactic plane (Reid \& Majewski 1993).

Several methods have been used for analyzing the stellar density distribution of the thick disc. Most of them are focused in identifying a specific population that can be used as tracer of the population of the thick disc. Sandage (1987), Freil (1987), Casertano et al. (1990), or Bergeat et al. (2002) used studies based on kinematic measurements, but there are also works that use star counts and Galactic model distributions, together with proper motions (Ojha et al. 1994a,b, 1996) or with colour distribution analyses (Yamagata \& Yoshii 1992; Robin et al. 1996; Haywood et al. 1997; Ng et al. 1997; Buser et al. 1999; Ojha 2001; Siegel et al. 2002). The general parameters derived for this component are not still well defined. The density of the thick disc has been usually defined as a double exponential, in the same way as for the thin disc, with a radial scalelength in the range of $2.5-4.5 \mathrm{kpc}$, a vertical scaleheight of roughly $700-2000 \mathrm{pc}$, and a local density of 2-10\% of the thin disc density (Reid \& Majewski 1993; Spagna et al. 1996; Robin et al. 1996; Ng et al. 1997; Buser et al. 1999; Ojha et al. 2001; Siegel et al. 2002; Larsen \& Humphreys 2003). In this paper, we have selected a different tracer for analyzing the vertical distribution in the Galactic disc: the red clump (RC) population. By using a simple empirical method of extracting the stellar density from Near Infrared colour-magnitude diagrams (NIR CMDs) we study the vertical distribution of the density of the RC giants.

This paper is organized as follows. In Sect. 2 we describe the method of obtaining the stellar density from the CMDs, and the density extracted in the direction of the Galactic poles using the method is analyzed in Sect. 3. The analysis of the vertical distribution of the thick disc component is extended to other ranges of latitudes in Sect. 4, while in Sect. 5 we analyze the radial variation of the density of the thick disc, obtaining the radial scalelength for this component. Finally, a summary with the main structural parameters of the thick disc and the further work necessary to continue the analysis of this Galactic component are described in Sect. 6.

The data for this work has been taken from the All Sky Release of the 2MASS project (Skrutskie et al. 1997, 
http: //www . ipac. caltech. edu/2mass), with a $100 \%$ coverage of the sky in the $J, H$ and $K_{\mathrm{s}}$ bands. We have made use of all available data for well off-plane regions of the survey $(|b|>$ $25^{\circ}$ ), where the nominal limiting magnitudes of the Survey can be reached $\left(J=15.8, H=15.1, K_{\mathrm{s}}=14.3\right)$. In this paper, we are assuming a value of $R_{\odot}=7.9 \mathrm{kpc}$.

\section{Stellar density from NIR CMDs}

An empirical method of obtaining both the interstellar extinction and the stellar density along a given line of sight was presented in López-Corredoira et al. 2002 (hereafter L02) and developed in subsequent papers (Picaud et al. 2003; Drimmel et al. 2003; López-Corredoira et al. 2004, hereafter L04).

The method is based on extracting the population of $\mathrm{K} 2 \mathrm{gi}-$ ants of the disc, that we asign to the RC stars, as they are the dominant giant population for this Galactic component (Cohen et al. 2000; Hammersley et al. 2000) and they are easily isolated in the CMDs. It was noted since long ago (Stanek et al. 1997; Paczynski \& Stanek 1998) that these stars form a conspicuous feature in the CMDs, which make them readily identifiable. The absolute magnitude, $M_{K}$ and intrinsic colour $(J-K)_{0}$ of this population is well defined: $M_{K}=-1.62 \pm 0.03,(J-K)_{0}=$ $0.61 \pm 0.01$, with a small dependence with the metallicity and age (Alves 2000; Grocholsky \& Sarajedini 2002; Salaris \& Girardi 2002; Pietrzyński et al. 2003). The exact identification of the spectral type is not important for the calculation, but the absolute magnitude and colour do matter. Indeed, some dispersion with respect these values is expected, but we know that this population is dominant and the dispersion of absolute magnitudes and colours is not very large so the use of an average value of $M_{K}$ and $(J-K)_{0}$ is a good approximation. This makes it possible to extract spatial information directly from the apparent magnitudes and $(J-K)$ colours of the RC stars in the colour-magnitude diagrams. (For a detailed description of the method, and the possible uncertainties in extracting the stellar density, see Sect. 3 in L02.)

In order to analyze the thick disc component in the Galaxy, we have examined $1 \times 1$ degree fields in the 2 MASS catalog within $15^{\circ}$ of the Galactic poles and apply the method to the resulting CMD (Fig. 1). We need to average several fields in order to obtain a sufficient number of RC stars or, in other words, to build up a conspicuous feature in the CMD which could be then easily identifiable. As shown in Fig. 1, the RC stars are easily identified in the CMD, forming an almost vertical stripe at $(J-K)=0.65$ due to the low extinction. With a dereddened colour of $(J-K)_{0}=0.61$, and the interstellar extinction value for $A_{K}$ as given by Rieke \& Lebofsky (1985), we obtain $A_{K} \sim 0.02 \mathrm{mag}$ for the selected fields.

In order to minimize the dwarf contamination in the counts, we have extracted stars for $m_{K}<10$. This limit is brighter than the one considered in L02 $\left(m_{K}<13\right)$ due to the lower extinction for the fields studied here. A higher extinction displaces the RC stars in the CMDs to redder colours as their apparent magnitude becomes fainter, helping to separate the dwarf and giant populations at fainter magnitudes. A value of $m_{K}=10$ corresponds to a distance from the Sun of $2.5 \mathrm{kpc}$ for a RC star, which is, therefore, suitable for analyzing the
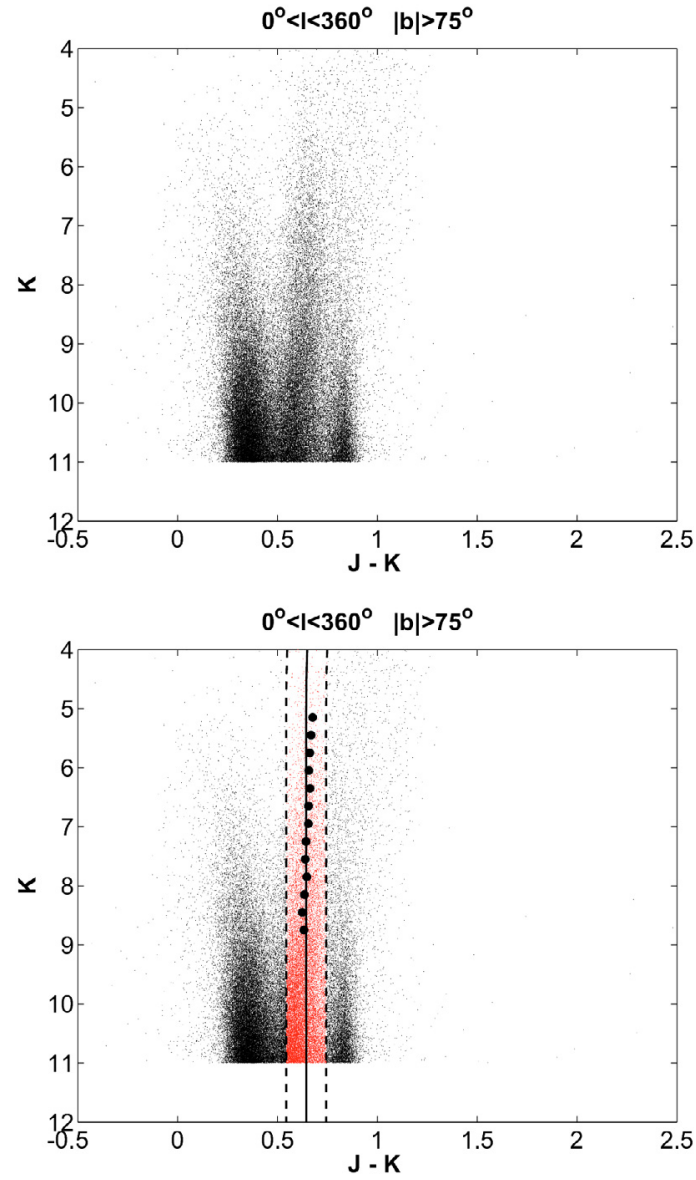

Fig. 1. Above: $K / J-K \mathrm{CMD}$ obtained combining all the 2MASS fields in $15^{\circ}$ around the Galactic poles, covering a total area of around $1500 \mathrm{deg}^{2}$. Below: $K / J-K \mathrm{CMD}$ with the fitted trace that we assign to RC giants population and the limits for the extraction of the stars (see Sect. 4 in Drimmel et al. 2003 for details).

disc more than $1 \mathrm{kpc}$ above the Galactic plane. A more complete analysis of the contamination by other populations in the counts is given in Sects. 3.2 and 3.3.

\section{A double exponential distribution for the stellar density}

The stellar density for the thin (TN) and thick (TK) disc can be represented as a double exponential, both respect to the galactocentric distance, $R$, and to the height above the Galactic plane, $z$ :

$\rho_{\mathrm{THIN}}(R, z)=\rho_{\odot, \mathrm{TN}} \exp \left(-\frac{R-R_{\odot}}{h_{R, \mathrm{TN}}}\right) \exp \left(-\frac{|z|}{h_{z, \mathrm{TN}}}\right)$

$\rho_{\mathrm{THICK}}(R, z)=\rho_{\odot, \mathrm{TK}} \exp \left(-\frac{R-R_{\odot}}{h_{R, \mathrm{TK}}}\right) \exp \left(-\frac{|z|}{h_{z, \mathrm{TK}}}\right)$.

The values given for the scalelengths of both components cover a wide range. For the thin disc, measurements in the NIR provide estimates in the range of 2-3 kpc (see, e.g., Ruphy et al. 1996; Drimmel \& Spergel 2001; Ojha 2001; L02). Scalelength of the thick disc is less well defined than that of the thin disc, with values from 2.5 to $4.5 \mathrm{kpc}$ (see Table 5 in 


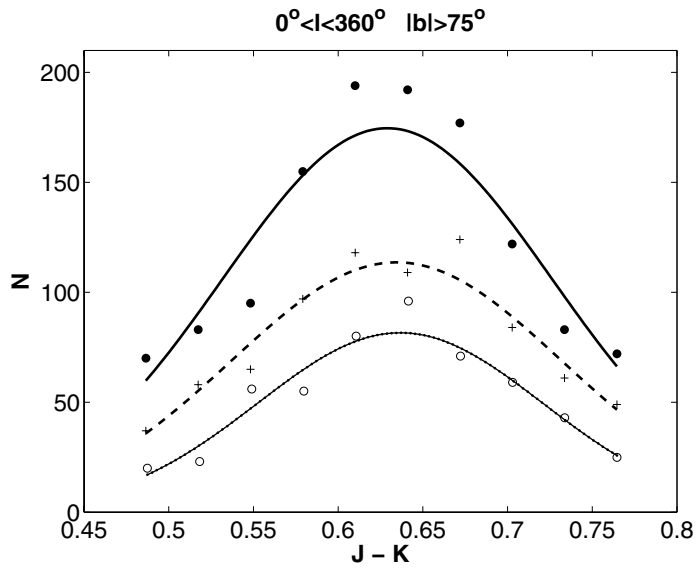

Fig. 2. Histograms of counts (per unit colour) for three fixed apparent magnitudes and the corresponding best Gaussian fits: $m_{K}=$ 9.85 (dots/solid line), $m_{K}=9.25$ (crosses/dashed line), $m_{K}=$ 8.65 circles/dot-dashed line. $\Delta m=0.3$.

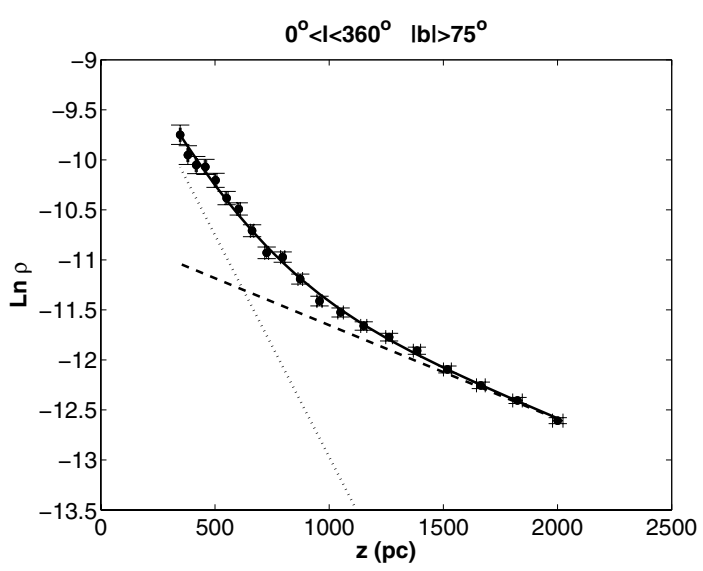

Fig. 3. Density of RC stars obtained from the CMD. The best fit for a sum of thick disc (dashed line) and thin disc (dotted line) components is also shown as a solid line. Error bars come from the Poisson noise in the extracted counts and from the uncertainty in the distance above the plane.

Buser et al. (1999) for a review of the thick disc scalelength and scaleheight measurements).

For the fields considered here $\left(|b|>75^{\circ}\right)$, the galactocentric distance covers a very narrow range between 7.8 and $8.6 \mathrm{kpc}$. So, we can consider the radial terms in Eqs. (1)-(2) nearly constant, and hence independent of scalelength. Using this, we can transform the density obtained from the diagrams to density with respect to the height above the Galactic plane.

In Fig. 3 we present the density of RC giants, obtained from the CMD, with respect to the height above the Galactic plane. As shown, a single exponential gives a poorer fit to the measured distribution. However, a double exponential provides an excellent fit, with one component dominating at distances up to $500 \mathrm{pc}$ above the Galactic plane and a second one dominating for distances larger than $1000 \mathrm{pc}$.

The sum of a thin disc and a thick disc components, each one with a double exponential distribution provides an accurate fit to the observed distribution. The analytical expressions

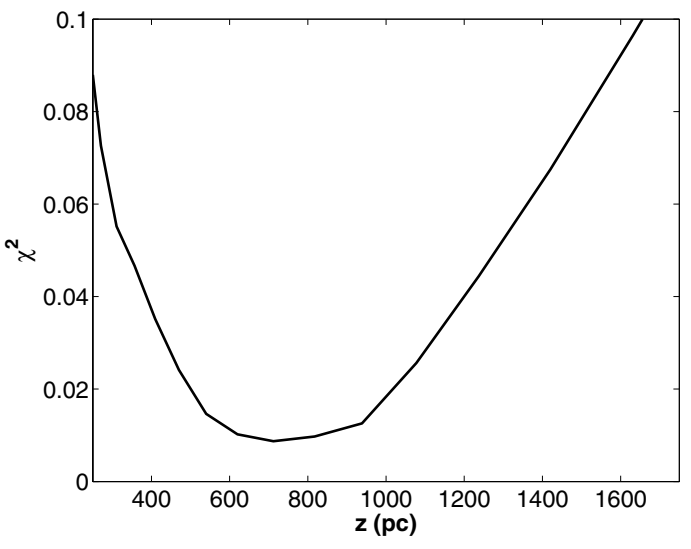

Fig. 4. $\chi^{2}$ vs. $z$ of double exponential fit of the RC star density.

obtained for those distributions are:

$\rho_{\text {RC,THIN }}(z)=(1.96 \pm 0.23) \times 10^{-4} \exp \left(\frac{-|z|}{225.41 \pm 27.98}\right)$

$\rho_{\mathrm{RC}, \mathrm{THICK}}(z)=(2.24 \pm 0.57) \times 10^{-5} \exp \left(\frac{-|z|}{1065.1 \pm 110.24}\right)$

with the density in stars $\mathrm{pc}^{-3}$. Those expressions have been obtained by using an iterative fitting to the observed density, without any preliminary assumption respect to the distances where each component is expected to be predominant (Fig. 4).

The resulting vertical scaleheight for the thin disc is consistent with those from the literature, being in the canonical range of 250-350 pc (Bahcall \& Soneira 1984; Robin \& Crézé 1986; Reid \& Majewski 1993; Siegel et al. 2002; L02). The scaleheight of the thick disc we obtained is also in agreement with other studies based on visible star count data and proper motions, with values in the range 910-1137 pc (Spagna et al. 1996; Ng et al. 1997; Buser et al. 1999; Larsen \& Humphreys 2003). Other works give shorter values: $760 \pm 50 \mathrm{pc}$ (Robin et al. 1996), $860 \pm 200 \mathrm{pc}$ (Ojha et al. 2001), 700-900 pc (Siegel et al. 2002), but not very far from ours and always in the range of 700-2000 pc described by Reid \& Majewski (1993). The wide range values cited above is another demonstration that how uncertain are still the structural parameters of this component.

\subsection{Metallicity effect in the $R C$}

When dealing with photometric parallaxes it is necessary to have well constrained values for both the absolute magnitude and intrinsic colour of the population used as distance estimator. The absolute magnitude and intrinsic colour considered here for the RC population are well established by previous studies (Alves 2000; Grocholsky \& Sarajedini 2002; Salaris \& Girardi 2002; Pietrzyński et al. 2003). In L02 and L04, the RC population has been used for extracting densities for the thin disc component, where no large metallicity gradient is expected (Cameron 1985; Sarajedini et al. 1995), and the range of ages and metallicities for the young disc component do not affect substantially the values for $M_{K}$ and $(J-K)_{0}$ of the RC. The thick disc component is an older and lower 
metallicity component (usually considered that of the 47 Tuc: $[\mathrm{Fe} / \mathrm{H}]=-0.7$ dex (Armandroff 1989; Liu \& Chaboyer 2000). The theoretical predictions of Salaris \& Girardi (2002) about the dependence of $M_{K}$ for the RC population with the age and metallicity give a difference of about -0.2 mag respect to the $\mathrm{RC}$ absolute magnitude in the solar neighborhood (Salaris \& Girardi 2002, Fig. 3). This difference means a distance uncertainty of $\sim 10 \%$, although the accuracy of the predictions of the models is 0.1-0.15 mag (Pietrzyński \& Gieren 2002; Pietrzyński et al. 2003). Using this, we can estimate an absolute magnitude of $M_{K}=-1.55 \pm 0.05$ with the metallicity assumed for the thick disc, in order to verify the compatibility of the results of the predictions for the model and the observed distribution (although we always prefer to follow the observational trend better than the predictions of any model). Repeating the analysis (with the assumption of an absolute magnitude for the $\mathrm{RC}$ of $\left.M_{K}=-1.55\right)$ the results are very similar to those of Eq. (3) with differences in the parameters of $\sim 4 \%$, a percentage that is included within the error bars. These estimates support the assumption that the absolute magnitude of the RC considered here does not change noticeably with the metallicity, and if it changes, it does within the errors of the parameters.

$\rho_{\text {RC,THIN }}(z)=(1.54 \pm 0.47) \times 10^{-4} \exp \left(\frac{-|z|}{255.3 \pm 16.37}\right)$

$\rho_{\text {RC,THICK }}(z)=(1.98 \pm 0.25) \times 10^{-5} \exp \left(\frac{-|z|}{1115.7 \pm 74.9}\right)$.

A more tricky question is the selected colour interval for the extraction of RC stars. The RC absolute magnitude in $J$ is more sensitive to metallicity and age than the $K$ filter, hence the intrinsic colour $(J-K)_{0}$ depends on both of metallicity and age (Salaris \& Girardi 2002; Grocholski \& Sarajedini 2002; Pietryński et al. 2003). The colour $(J-K)_{0}$ considered for the RC population has no strong influence in the results, as we can assume an approximately zero extinction ( $\sim 0.02 \mathrm{mag}$ as obtained from the CMD in Fig. 1). Therefore, the observed $(J-K)$ for the RC stars is nearly the value corresponding to the intrinsic colour $(J-K)_{0}$ for this population. However, the colour interval used for extracting the RC population might exclude a significant fraction of stars with metallicities lower than $[\mathrm{Fe} / \mathrm{H}]=-0.8$ dex, which has a de-reddened colour $(J-K)_{0}$ around 0.5 . This value, when translated to the measured colour will be around $0.53 \pm 0.03$, just in the lower limit of the extraction. By a direct visual inspection of the CMD and the colour histograms obtained, both for the approximated RC colour range (Fig. 5) and for the whole set of data (Fig. 6) we observe that the maximum of the population is well defined and there is a relative low number of stars with $(J-K)$ colour very far from the bulk of the distribution of stars, well centered around $(J-K) \sim 0.65 \pm 0.1 \mathrm{mag}$. This results suggests that the relative abundance of these low metallicity stars is not that high, hence they do not affect to the extracted densities. In the case they were an important contribution to the counts, some feature in the blue side of the colour histogram should emerge, which is not the case (see Fig. 5).

Even so, we can repeat the extraction with a higher colour interval, $0.2 \mathrm{mag}$ instead of $0.1 \mathrm{mag}$ in order to accommodate

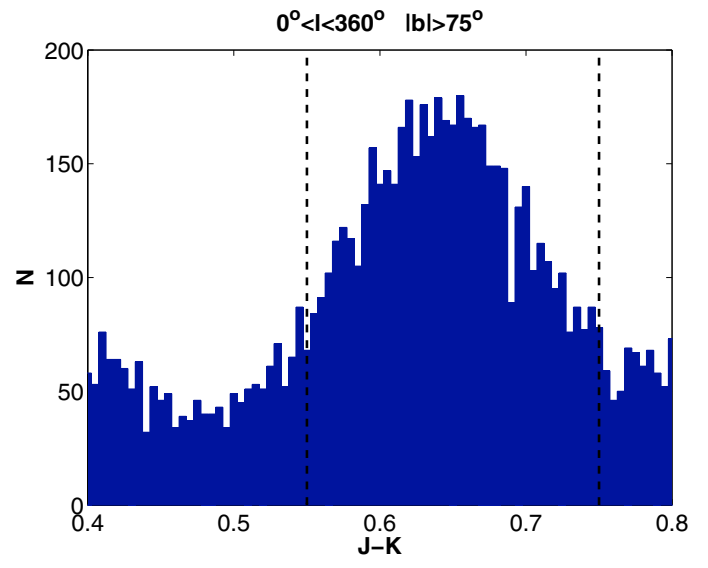

Fig. 5. Number counts respect to the colour $(J-K)$, for the region corresponding to the $\mathrm{RC}$ population. The lines show the colour interval selected for extracting the RC stars.

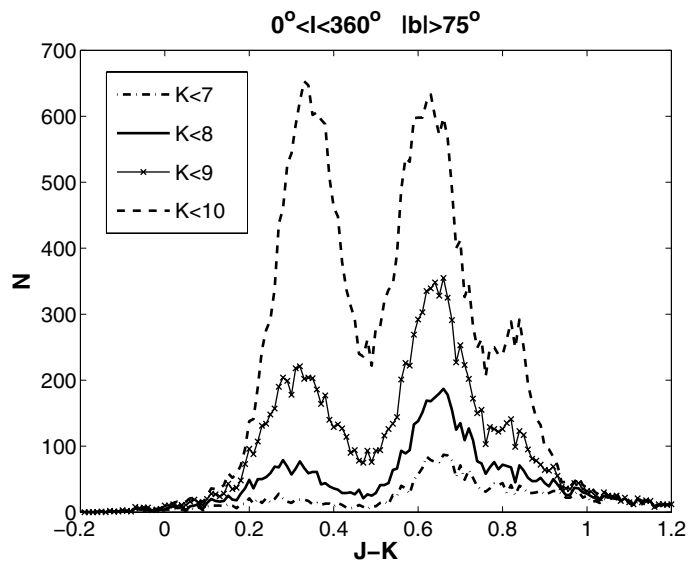

Fig. 6. Colour distribution of the stars in the fields $|b|>75^{\circ}$ up to different limiting magnitudes. The proportion of other giants respect to the RC population decreases when the limiting magnitude for the extraction becomes fainter.

for the stars in the blue side. We obtain that for $\Delta(J-K)=$ 0.2 mag the contamination by dwarf stars is nearly the same than for $\Delta(J-K)=0.1 \mathrm{mag}$ (Sect. 3.2), so the results must not be affected by this. We derived a density well fitted by a double exponential (Fig. 7), with similar parameters than before:

$$
\begin{aligned}
& \rho_{\text {RC,THIN }}(z)=(2.31 \pm 0.55) \times 10^{-4} \exp \left(\frac{-|z|}{215.1 \pm 17.88}\right) \\
& \rho_{\text {RC,THICK }}(z)=(2.63 \pm 0.33) \times 10^{-5} \exp \left(\frac{-|z|}{1216.1 \pm 86.4}\right) .
\end{aligned}
$$

If the colour interval for the extraction of the RC stars would be affecting to the extracted densities as a result of a metallicity change that alters to the RC absolute magnitude and intrinsic colour, one would expect a discrepant result than the previous one, and it is not the case. Moreover, when the interval increases to $\Delta(J-K)=0.3$ mag the results became very confused and unrealistic, by the mixture of different stars (dwarfs, $\mathrm{M}$ giants, etc.) that can not be assumed to be RC population. This result is also compatible with the conclusions obtained in Sect. 3.2.2 of L04, where the relative dependence of the colour interval used for determining the density was discussed. 


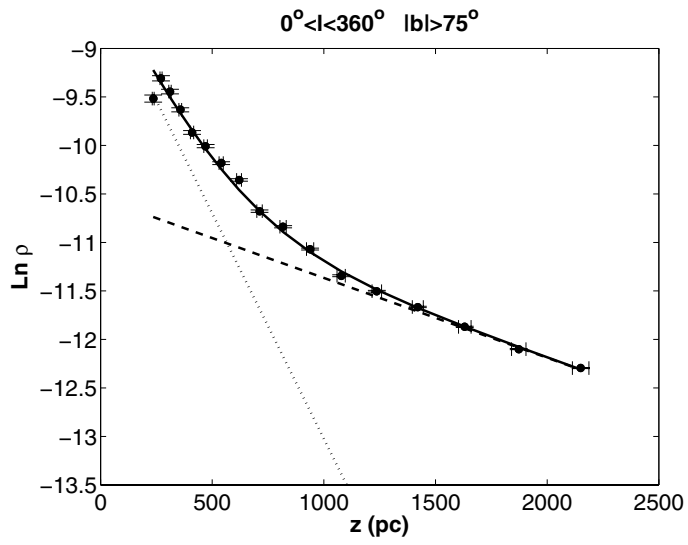

Fig. 7. Same as Fig. 3, but with the density obtained after using a colour interval of 0.2 mag for the extraction of the RC stars.

\subsection{Dwarf contamination}

One of the main difficulties in obtaining the RC density from the CMD is isolating that stellar component from others that contaminated the counts. The extent of the contamination of the $\mathrm{K}$ giants is more severe at the blue side, and at fainter magnitudes when the RC stripe mixes with the dwarfs (see Fig. 1). As already noted, when too many dwarf stars are included in the counts, but they are assumed to be RC stars, then the extracted density would be overestimated at larger $z$, producing the same effect than an additional component that dominates the counts. Hence we must be very careful in minimizing the contamination of dwarf stars in the extracted density.

To estimate the possible dwarf contamination in the extracted counts we use the luminosity and densities functions of the giants and dwarf stars as defined in the model by Wainscoat et al. (1992). We have calculated the predicted ratio between dwarfs and the total of giants plus dwarfs in the counts. The result is shown in Fig. 8. It is clear that for faint apparent magnitudes $\left(m_{K} \simeq 11.5\right)$ the dwarf contribution is important, with almost $50 \%$ of the total counts. However, the contribution decreases for lower apparent magnitudes, with less than $\sim 10 \%$ of dwarf stars for $m_{K}<10$. Here we will use a limiting magnitude of $m_{K}=10$ for the extracted stars, avoiding the possible contamination of dwarf stars in the density of RC stars.

The method assumes that once the trace of the RC population has been obtained, all the stars within a fixed interval of colour $(J-K)$ around the trace are RC stars. This range has usually been considered as $\Delta(J-K)=0.2 \mathrm{mag}$, because it produces the best compromise between avoiding contamination of other population without losing too many RC stars (see Sect. 3.3 in L02 and Sect. 3.1 in L04 for a detailed description of the dependence of the extracted density with respect to the colour interval used in the extraction). In the regions analyzed in this paper, the interstellar extinction is negligible, as we are looking far away the Galactic plane. That produces an almost vertical RC stripe and narrower that those of the Galactic plane areas. For this reason we can use an even narrower colour interval for the extraction, compared with that used in L02. This will minimise the dwarf contamination in the faint end. Hence we have restricted the colour interval for the extraction of the

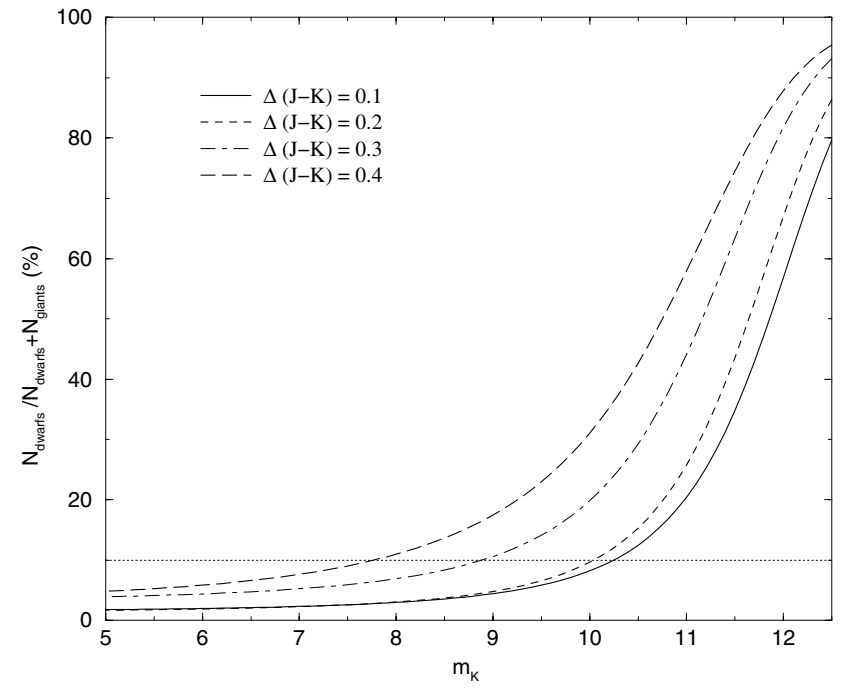

Fig. 8. Variation of the ratio of predicted dwarf stars with the magnitude $K$ in the RC extraction, for different colour intervals $\Delta(J-K)$. The contamination is less than $10 \%$ in the extraction of giant stars within $0.1 \mathrm{mag}$ around the fitted trace to the RC up to $m_{K}=10$.

$\mathrm{RC}$ stars to 0.1 mag around the fitted trace. The contamination on the red side of the giant stripe, largely due to $\mathrm{M}$ giants, is also less intense compared with the in-plane regions where the higher values of the extinction tends to mix this two populations. In Fig. 8 we also show the effect of increasing the colour interval for the extraction of the RC stars, $\Delta(J-K)$, on the dwarf contamination. As shown, the differences in setting the colour interval for the extractions, up to $m_{K}=10$, are significant when moving from 0.2 to 0.3 or higher. Between 0.2 and 0.1 the difference is not that large, but still 0.1 produces better results in the sense of less dwarf contamination. Because of this and to the fact that the total number of sources in the extracted RC stripes is virtually the same up to $m_{K}=9$, we have finally selected 0.1 as the colour interval used in the source selection.

Using this model we can correct the extracted density by subtracting the theoretical contribution of the dwarf stars. The density law obtained has two components, and a double exponential fit yields to very similar values than before:

$\rho_{\mathrm{RC}, \mathrm{THIN}}(z)=(1.94 \pm 0.24) \times 10^{-4} \exp \left(\frac{-|z|}{222.94 \pm 15.72}\right)$

$\rho_{\mathrm{RC}, \mathrm{THICK}}(z)=(2.27 \pm 0.59) \times 10^{-5} \exp \left(\frac{-|z|}{1014.65 \pm 100.44}\right)$

in stars $\mathrm{pc}^{-3}$. With this result, we can conclude that the effect of the dwarf contamination in the counts is not enough to explain the excess in the density for heights larger than $800 \mathrm{pc}$ observed in the vertical distribution of the density of the RC stars, as we are avoiding of this effect by extracting the RC stars in a very narrow range of colour around the fitted trace, $\Delta(J-K)=0.1$, and extracting stars up to $m_{K}<10$ when the contamination becomes important (see Fig. 1). 


\subsection{Contamination by giants}

Apart from the dwarfs there is also the possibility of contamination from giants which have the same colour as the RC but have a different absolute magnitude. The giants and supergiants that are more luminous than the $\mathrm{RC}$ are not an issue here as these would appear to have a far shorter scaleheight, when the method is applied to them. The stars which have a luminosity slightly lower luminosity cannot be as easily dismissed, however. In principle, if there were a significant number of stars about 2 mag fainter than the $\mathrm{RC}$ these would produce the same effect on the counts as would the thick disc. In order to explore this possibility we have attempted to determine the luminosity function for the range of $(J-K)$ sources being used for the analysis. Ideally this could be done by combining the parallexes determined from Hipparcos with the $K$ magnitudes from 2MASS for the stars with $(J-K)$ colour being used for this study. Unfortunately, the maximum distance to which reliable parallaxes exists (i.e. error of $\sim 10 \%$ ) is about $150 \mathrm{pc}$, but at this distance the RC stars are too close to saturation in 2MASS to be reliable. Therefore, we have used the MSX A band data (hereafter [8.3]) which is complete brighter than [8.3] $=6.1$ mag. The [8.3] $-K$ colour for photospheric stars (as is generally the case here) is only about 0.1 and so the luminosity function for [8.3] will be very similar to that at $K$. The MSX and Point source catalogue and the Hipparcos Output Catalogue (Hoeg et al. 1997) were cross-correlated (Cohen et al. 2000) and the sources with a [8.3] $<6$ and $2.3<V_{\text {Tycho }}-[8.3]<2.9$ were selected (see Fig. 4 of Cohen et al. 2000). The absolute [8.3] magnitude was then calculated using the measured parallax for those source closer than $120 \mathrm{pc}$. The histogram of the absolute magnitudes was then divided by the volume in which each magnitude would be seen to give the space density (see Fig. 9). No attempt has been made to correct for extinction as at 8 microns the effect is negligible over these distances. Also Malmquist and Kulz-Kelker biases were ignored as these would effect both the RC and lower luminosity giants in a similar fashion.

The determined luminosity function was then used in a Monte Carlo simulation which predicted the star counts in the range $b>75^{\circ}$ assuming a single exponential with a scaleheight of $300 \mathrm{pc}$. The [8.3] counts were converted into $K$ counts by adding $0.1 \mathrm{mag}$. The predicted counts were nomalised to the measured counts at $m_{K}=+8$. Figure 10 shows the predicted counts (crosses) and the measured counts (dots). As can be seen there is reasonable agreement between the measured and predicted counts for source brighter than $m_{K}=+8.5$, but the predicted counts flatten at $m_{K}=9$ whereas the real counts continue to rise. The predicted counts do not begin to rise again until $m_{K}=12$ when the dwarfs become the dominant source type. When the counts are converted into a plot of log density versus distance using the method described previously in Sect. 2 (see Fig. 11), the predicted counts follow an almost straight line until nearly $2 \mathrm{kpc}$, whereas the real counts begin to diverge from the straight line beyond about $750 \mathrm{pc}$. It is possible that some of the difference between the predicted and measured counts is due to errors in the luminosity function, however at $m_{K}=10$ the model with a single exponential is predicting only $30 \%$ of

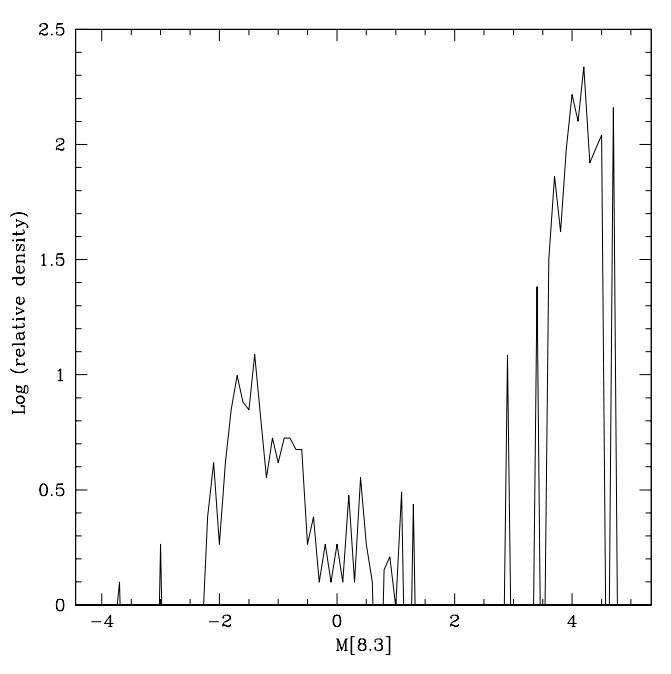

Fig. 9. $M_{[8.3]}$ luminosity function for those sources with the same $V_{\text {Tycho }}-[8.3]$ colour as the RC. The RC sources have an $M_{[8.3]}$ of about -1.7 and the lower luminosity continue to $M_{[8.3]}=1$. There are then no stars until the dwarfs at around $M_{[8.3]}=4$.

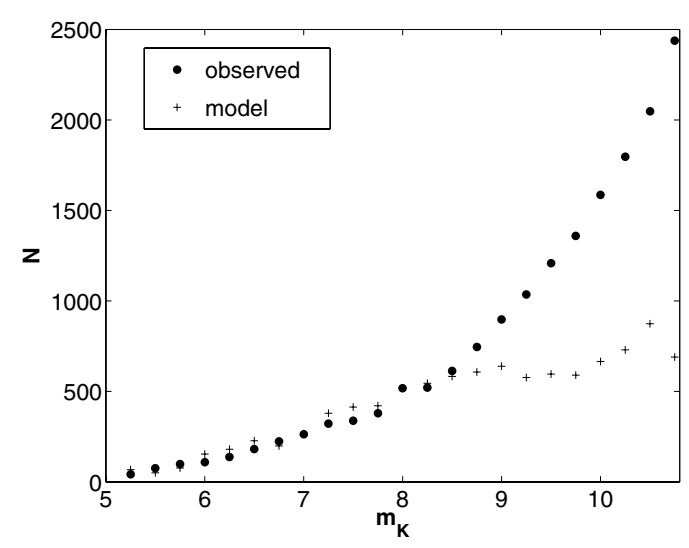

Fig. 10. Comparison between the predicted counts obtained with the Monte Carlo simulation (crosses) and the observed counts (dots) extracted for the fields with $b>75^{\circ}$.

the measured counts. It would require an unreasonably large alteration to the luminosity function near $M_{K}=+1$ to bring the model counts into alignment with the measured counts. Hence, the luminosity function, in particular lower luminosity giants, cannot be the reason for the vertical density function apparently following a double rather than single exponential.

A second way of checking the effect that the low luminosity giants have in the counts, is determining the variation of the ratio of these stars respect to the $\mathrm{RC}$ stars with magnitude, in the same way as in Sect. 3.2. We use the empirical luminosity function obtained before with the MSX counts and Hipparcos parallaxes (Fig. 9) and compared the predicted number of these stars in the counts that we assume correspond to the RC population (Fig. 12). This shows that of the stars between $9<$ $m_{K}<10$, only $15 \%$ are the lower luminosity giants, a value that is of the same order as the dwarf contamination estimated in Sect. 3.2. For brighter apparent magnitudes, the contamination by this type of stars is less than $2.5 \%$, so it is unimportant. 


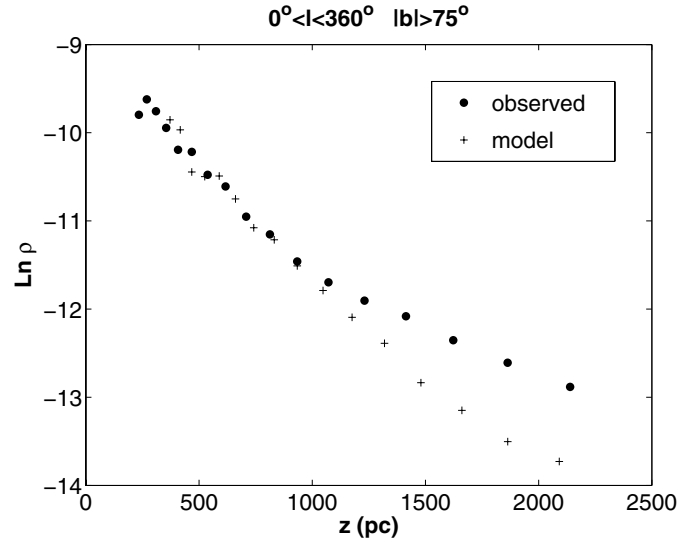

Fig. 11. Density of RC stars obtained from the CMD (dots) and that obtained from the simulation, corresponding to a single exponential with a scaleheight of $300 \mathrm{pc}$ (crosses).

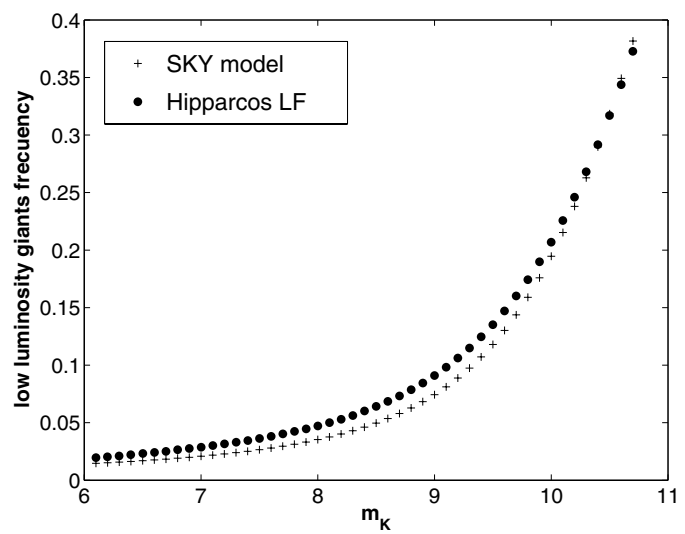

Fig. 12. Ratio of predicted low luminosity giant stars in the extracted RCpopulation, as in Fig. 8 for dwarf stars. The contamination is between $10 \%-20 \%$ for $9<m_{K}<10$, that corresponds to distances in the range $1.3<r<2.1 \mathrm{kpc}$ for a RC star. For comparison, we also show the predicted ratio obtained by using the SKY model instead of the empirical luminosity function of Hipparcos, without noticeably differences.

\subsection{Malmquist bias in the absolute magnitude of $R C$ stars}

In a magnitude limited sample, the brighter stars are statistically over-represented and the derived absolute magnitudes are too bright because the distance to which intrinsically bright stars are visible is larger than the corresponding one for faint stars. We can evaluate the effect of the so-called Malmquist bias (Malmquist 1920) for the case in which the luminosity function of the population is a Gaussian, by the general formula (Binney $\&$ Merrifield 1998):

$M(K)=M_{0}-\sigma^{2} \frac{\mathrm{d} \ln A(K)}{\mathrm{d} K}$,

where $M(K)$ is the assumed absolute magnitude, $M_{0}$ is the true absolute magnitude of the population, $\sigma$ is the dispersion of the luminosity function and $A(K)$ is the differential counts evaluated at the apparent magnitude $K$ of any star.

The dispersion in absolute magnitude for the obtained distribution of $\mathrm{RC}$ stars is around $\sim 0.1 \mathrm{mag}$, that produces a

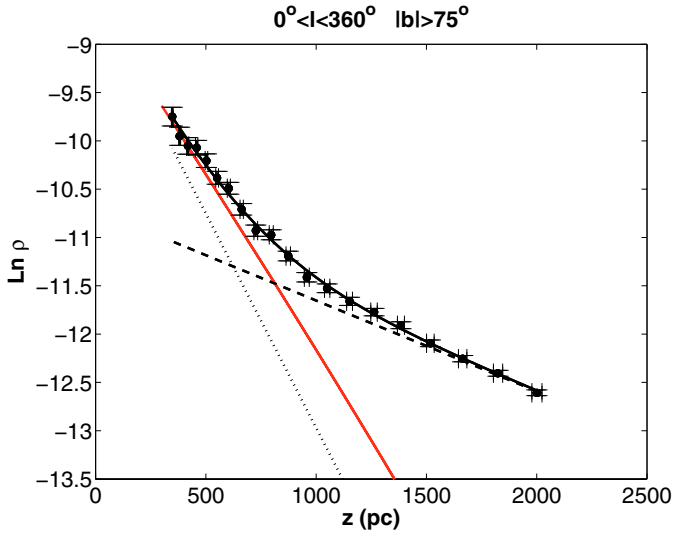

Fig. 13. Same as Fig. 3, now with predicted density by the model L02 is shown as the straight solid line. Up to 500 pc there is a good agreement between the predicted and the observed density, but for $z>800 \mathrm{pc}$ the predictions are clearly underestimating the observed vertical distribution.

correction due to the Malmquist bias of less than $0.01 \mathrm{mag}$, and negligible errors in the derived distances. The correction is independent of the apparent magnitudes considered in the analysis, as we are using only stars 5 mag brighter than the limiting magnitude of the survey, a range where the differential counts have a nearly constant gradient.

\subsection{Comparison with LO2 disc model predictions}

In L02 a disc model is fit to 2MASS star counts in the $K$ band, that includes the effects of a flare and a warp. With those data the observed vertical distribution of the counts were compared with the predictions of the model (which did not include a thick disc) and no conclusive remarks respect to the presence of the thick disc component were made, mainly due to the lack of 2MASS data at that time (see Sect. 6.3 in L02). Now, with a full sky coverage, we repeated the comparison more accurately. As Fig. 13 shown, the L02 model for the thin disc is only compatible with the observed density up to $500 \mathrm{pc}$ above the Galactic plane.

\section{Looking for the thick disc in other latitudes}

It is clear that the latitudes near to the Galactic poles are the best to analyze the presence of the thick disc. However, this component must also be found at other galactic latitudes when the contrast with the local disc component is strong enough. We have therefore repeated the analysis of Sect. 3 in other ranges of galactic latitudes. Again, we collected all the $1 \times 1$ degree 2MASS fields in three latitude intervals: $25^{\circ}<|b|<35^{\circ}$; $45^{\circ}<|b|<55^{\circ}$, and $55^{\circ}<|b|<75^{\circ}$. Now in the analysis we have also separated the data coming from the positive latitudes and for the negative ones, as the interstellar extinction is higher than in the case of the Galactic poles, and asymmetrically distributed above and below the Galactic plane. This may produce undesirable effects when the whole data are combined into an only CMD.

In Table 1 are summarized the parameters obtained for a double exponential fit to the resulting density extracted from 
Table 1. Parameters for the sum of thin and thick disc components.

\begin{tabular}{cccccc}
\hline \hline Range of $b$ & $\begin{array}{c}\text { Area } \\
{\left[\mathrm{deg}^{2}\right]}\end{array}$ & $\begin{array}{c}\rho_{\odot, \mathrm{THIN}} \\
{\left[\mathrm{stars} \mathrm{pc}^{-3}\right]}\end{array}$ & $\begin{array}{c}h_{z, \text { THIN }} \\
{[\mathrm{pc}]}\end{array}$ & $\begin{array}{c}\rho_{\odot, \mathrm{THICK}} \\
{\left[\mathrm{stars} \mathrm{pc}^{-3}\right]}\end{array}$ & $\begin{array}{c}h_{z, \text { THICK }} \\
{[\mathrm{pc}]}\end{array}$ \\
\hline $25^{\circ}<|b|<35^{\circ}$ & 6260 & $(1.57 \pm 0.53) \times 10^{-4}$ & $204.63 \pm 25.96$ & $(3.45 \pm 0.40) \times 10^{-5}$ & $820.99 \pm 102.63$ \\
$25^{\circ}<b<35^{\circ}$ & 3130 & $(2.49 \pm 1.57) \times 10^{-4}$ & $100.96 \pm 19.52$ & $(7.08 \pm 1.36) \times 10^{-5}$ & $522.69 \pm 52.41$ \\
$-25^{\circ}>b>-35^{\circ}$ & 3130 & $(1.69 \pm 0.97) \times 10^{-4}$ & $140.33 \pm 42.27$ & $(6.30 \pm 1.19) \times 10^{-5}$ & $599.37 \pm 53.91$ \\
$45^{\circ}<|b|<55^{\circ}$ & 4670 & $(1.48 \pm 0.82) \times 10^{-4}$ & $247.15 \pm 34.64$ & $(2.02 \pm 0.34) \times 10^{-5}$ & $1077.3 \pm 70.47$ \\
$45^{\circ}<b<55^{\circ}$ & 2335 & $(1.37 \pm 1.08) \times 10^{-4}$ & $230.64 \pm 26.53$ & $(2.42 \pm 0.53) \times 10^{-5}$ & $940.60 \pm 53.02$ \\
$-45^{\circ}>b>-55^{\circ}$ & 2335 & $(1.62 \pm 0.22) \times 10^{-4}$ & $256.72 \pm 28.91$ & $(1.73 \pm 0.34) \times 10^{-5}$ & $1239.6 \pm 134.13$ \\
$55^{\circ}<|b|<75^{\circ}$ & 6168 & $(1.48 \pm 0.46) \times 10^{-4}$ & $261.63 \pm 36.64$ & $(1.72 \pm 0.13) \times 10^{-5}$ & $1179.4 \pm 64.66$ \\
$55^{\circ}<b<75^{\circ}$ & 3084 & $(1.02 \pm 0.98) \times 10^{-4}$ & $314.05 \pm 48.83$ & $(1.38 \pm 0.28) \times 10^{-5}$ & $1255.8 \pm 124.85$ \\
$-55^{\circ}>b>-75^{\circ}$ & 3084 & $(2.15 \pm 0.16) \times 10^{-4}$ & $220.82 \pm 36.98$ & $(2.11 \pm 0.29) \times 10^{-5}$ & $1093.2 \pm 67.39$ \\
$|b|>75^{\circ}$ & 1500 & $(1.96 \pm 0.23) \times 10^{-4}$ & $225.41 \pm 27.98$ & $(2.24 \pm 0.57) \times 10^{-5}$ & $1065.1 \pm 110.24$ \\
$b>75^{\circ}$ & 750 & $(1.41 \pm 1.01) \times 10^{-4}$ & $259.37 \pm 27.34$ & $(1.87 \pm 0.71) \times 10^{-5}$ & $1135.3 \pm 118.04$ \\
$b<-75^{\circ}$ & 750 & $(2.75 \pm 0.46) \times 10^{-4}$ & $197.72 \pm 29.28$ & $(2.53 \pm 0.89) \times 10^{-5}$ & $1018.6 \pm 119.06$ \\
ALL DATA & 18598 & $(1.52 \pm 0.56) \times 10^{-4}$ & $248.27 \pm 12.92$ & $(1.93 \pm 0.19) \times 10^{-5}$ & $1132.2 \pm 45.22$ \\
\hline
\end{tabular}
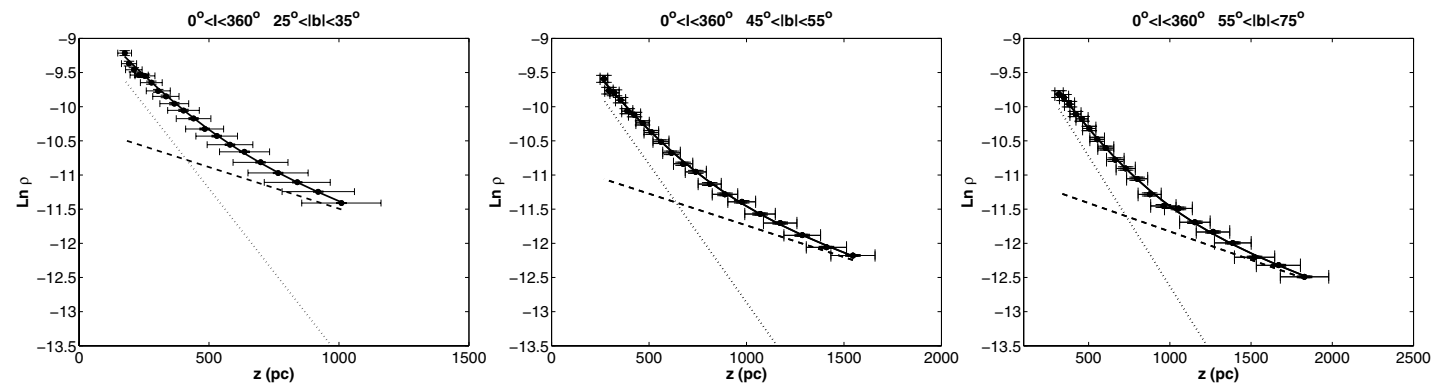

Fig. 14. Density of RC stars obtained from the CMDs and the best fit to a double exponential distribution in the fields: $25^{\circ}<|b|<35^{\circ}(l e f t)$, $45^{\circ}<|b|<55^{\circ}$ (centre) and $55^{\circ}<|b|<75^{\circ}$ (right). Symbols are the same than in Fig. 3 .

the CMDs and in Fig. 14 is shown the density extracted and the best fit for each range of galactic latitudes. For a global comparison we have also included the results of Sect. 3, also showing the results for both the Northern and Southern Galactic poles. These fits are obtained neglecting the radial dependence with $R$ in the density as given in Eqs.(1)-(2). It is clear that this can be assumed for the direction of the Galactic poles, as we cover very narrow range of $R$, however it is not so evident in the rest of directions (see Sect. 4.1).

For $45^{\circ}<|b|<55^{\circ}$ and $55^{\circ}<|b|<75^{\circ}$ there is a clear double trend in the extracted density, that supports the inclusion of a double exponential in the fit. However, for $25^{\circ}<|b|<35^{\circ}$ we obtain more discrepant results both for the thin and the thick disc. It may be due to the fact that for this galactic latitudes, we can only extract the stellar density up to $950 \mathrm{pc}$ from the Galactic plane, so we are not reaching the region where the thick disc component becomes predominant. Also, this is the galactic latitude interval where there are a higher uncertainty in the value of $z$ assigned to the RC giants. The iterative fit does not provide accurate results in this case and even a single exponential fit to the extracted density can be acceptable, with a scaleheight of 357.14 pc for this single component. Hence $25^{\circ}<|b|<35^{\circ}$ will not be used in this analysis. However, the results in the parameters for $45^{\circ}<|b|<55^{\circ}$ and $55^{\circ}<|b|<75^{\circ}$ are comparable to those obtained in Sect. 3, and are included in the expected range of values both for the thin and the thick disc. No significative differences are found between the results for the fields considering either positive

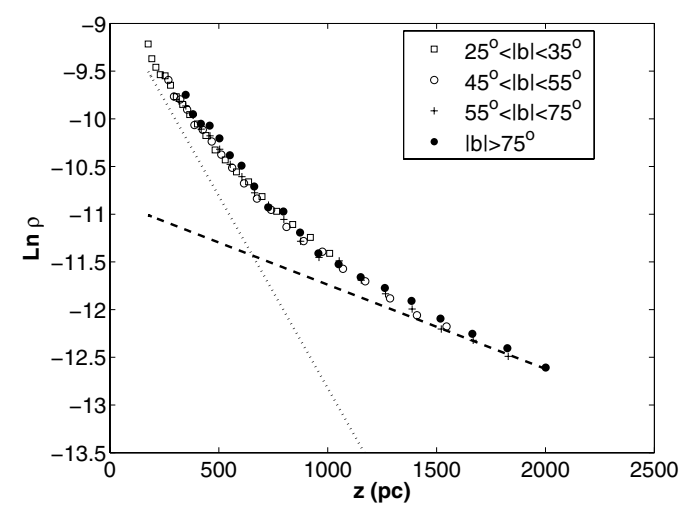

Fig. 15. Density distribution combining all the global results summarized in Table 1. The best fits both for the thin (dotted line) and thick disc (dashed line) components are shown.

or negative latitudes, once the correction for the distance of the Sun above the Galactic plane was included $\left(z_{\odot}=15 \mathrm{pc}\right.$; Hammersley et al. 1995), although some slightly variations due to extinction effects (that is higher for $b<0^{\circ}$ ) can be present in the results.

For each latitude range, the interval of $z$ covered is different due to by the limiting magnitude used for the extraction of RC stars (see Sect. 3.2). This allows us to improve the fit by considering all the results as a whole. The result of combining all the densities obtained for the different latitude ranges is shown in Fig. 15 and the parameters obtained are 

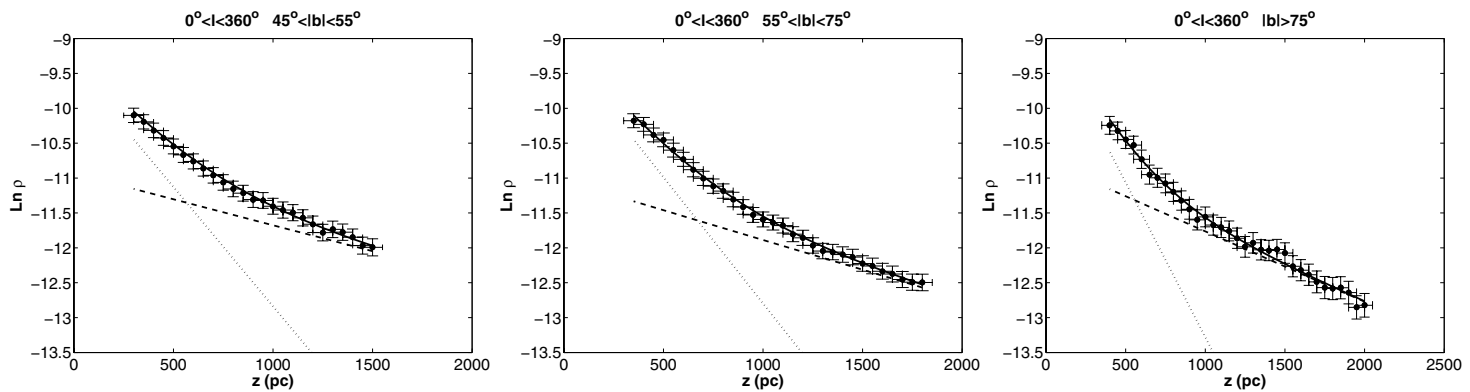

Fig. 16. Density of RC stars obtained from the CMDs and the best fit to a double exponential distribution in the fields: $45^{\circ}<|b|<55^{\circ}(l e f t)$, $55^{\circ}<|b|<75^{\circ}$ (centre) and $|b|>75^{\circ}$ (right), selecting only those stars with galactocentric distances in the range $7.65 \mathrm{kpc}<R<8.15 \mathrm{kpc}$, except for $|b|>75^{\circ}$, where the extracted stars have $R=7.9 \pm 0.1 \mathrm{kpc}$. Symbols are the same than in Figs. 3 and 14.

Table 2. Parameters for the sum of thin and thick disc components, when the range of $R$ has been restricted.

\begin{tabular}{cccccc}
\hline \hline Range of $b$ & $\begin{array}{c}\text { Area } \\
{\left[\mathrm{deg}^{2}\right]}\end{array}$ & $\begin{array}{c}\rho_{\odot, \mathrm{THIN}} \\
{\left[\mathrm{stars} \mathrm{pc}^{-3}\right]}\end{array}$ & $\begin{array}{c}h_{z, \text { THIN }} \\
{[\mathrm{pc}]}\end{array}$ & $\begin{array}{c}\rho_{\odot, \mathrm{THICK}} \\
{\left[\mathrm{stars} \mathrm{pc}^{-3}\right]}\end{array}$ & $\begin{array}{c}h_{z, \text { THICK }} \\
{[\mathrm{pc}]}\end{array}$ \\
\hline $45^{\circ}<|b|<55^{\circ}$ & 4670 & $(0.82 \pm 1.35) \times 10^{-4}$ & $292.34 \pm 39.28$ & $(1.78 \pm 0.54) \times 10^{-5}$ & $1143.3 \pm 167.22$ \\
$55^{\circ}<|b|<75^{\circ}$ & 6168 & $(1.03 \pm 0.38) \times 10^{-4}$ & $276.51 \pm 14.98$ & $(1.61 \pm 0.52) \times 10^{-5}$ & $1173.2 \pm 94.64$ \\
$|b|>75^{\circ}$ & 1500 & $(1.43 \pm 0.89) \times 10^{-4}$ & $225.58 \pm 29.52$ & $(2.13 \pm 0.63) \times 10^{-5}$ & $992.18 \pm 67.41$ \\
\hline
\end{tabular}

shown in Table 1 . We also include the density extracted for $25^{\circ}<|b|<35^{\circ}$ because they provide the best estimates for the lower heights above the plane and to show the values are in agreement with the results for other latitudes.

\subsection{The radial dependence}

In Sect. 3 we ignored the effect of the radial dependence in the density to extract the variation of the stellar density with the height. This can be done due to the narrow range of galactocentric distances covered when observing in the Galactic poles direction. For the rest of ranges considered in Sect. 4, the effect of this radial dependence of the density is not as negligible. We repeated the calculations of Sect. 4 taking into account this effect. With this purpose, the extracted stars were selected by the range in galactocentric distances in order to restrict the values of $R$ in Eqs. (1)-(2). For the comparison, we have only taken sources with $7.65 \mathrm{kpc}<R<8.15 \mathrm{kpc}$ (more or less symmetrically distributed around the value of $R_{\odot}$ ), so the results can be compared directly. For fields in the Galactic poles, we have been even more restrictive, selecting only those stars with $R=$ $7.9 \pm 0.1 \mathrm{kpc}$, that coincides with the typical value of $R_{\odot}$ assumed in L02 and L04. Fields with $25^{\circ}<|b|<35^{\circ}$ will be not considered here as this range of galactic latitudes is not appropriate for analyzing the thick disc component as it was discussed in the previous section.

Once again, a double exponential fit is required in the three cases. The parameters for the thin and thick disc components are in good agreement, with a weighted mean results for the scaleheights of $h_{z, \text { THIN }}=268.81 \pm 12.64 \mathrm{pc}$ and $h_{z, \text { THICK }}=$ $1061.9 \pm 52.16 \mathrm{pc}$, well within the expected range of values (Reid \& Majewski 1993; Spagna et al. 1996; Robin et al. 1996; $\mathrm{Ng}$ et al. 1997; Buser et al. 1999; Ojha et al. 2001; Siegel et al. 2002; Larsen \& Humphreys 2003). We will consider these values as representative of the thin and thick disc components, because they are more realistic than the obtained in Sect. 4, which are more affected by the uncertainties in the method. In any case, they are not very far (as expected) than the values summarized in Table 1 independent of the range of latitudes used (except for $25^{\circ}<|b|<35^{\circ}$ ). The contrast between the thin and thick disc in the Solar vicinity would be $\rho_{\odot, \mathrm{THICK}} / \rho_{\odot, \mathrm{THIN}}=$ $11.53 \% \pm 5.6 \%$.

\section{Radial scalelength of the thick disc}

In Sect. 4 we obtained the variation of the density with the height above the plane by assuming a narrow range of galactocentric distances to make the first term in Eqs. (1)-(2) negligible. We can now invert the process, and analyze the radial variation of the density once the vertical scaleheight has been obtained. In this way, we analyze 15 fields of 20 degrees wide in longitude with $45^{\circ}<|b|<55^{\circ}$. We select this range of latitudes because they provide a larger interval of galactocentric distances, with enough stars to obtain accurate estimates of the density. In each field, $K$ giant stars at heights larger than $1 \mathrm{kpc}$ above or below the Galactic plane are extracted, and the density respect to the galactocentric distance is then obtained using:

$\rho_{\mathrm{RC}}(R) \equiv \frac{\rho_{\mathrm{RC}}(R, z)}{\mathrm{e}^{\frac{-|z|}{h_{z}}}}=\rho_{\odot} \mathrm{e}^{\frac{-\left(R-R_{\odot}\right)}{h_{R}}}$.

As only stars far away from the plane are used, we assume that all the $K$ giant stars we are studying correspond to the thick disc component, so their radial variation is a reflect of the radial variation of the thick disc (observing the right panel in Fig. 14 it is shown that more than $92 \%$ of stars with $z>1 \mathrm{kpc}$ correspond to this component). The result is shown in Fig. 17, where the best fit is for: $\rho_{\odot, \text { THICK }}=(1.43 \pm 0.68) \times 10^{-5}$ stars $\mathrm{pc}^{-3}$ and $h_{R \text {,THICK }}=3.04 \pm 0.11 \mathrm{kpc}$. No significative differences are found in those parameters if we extract the stars at higher values of $z$ (see Table 3). Also, no differences have been found 


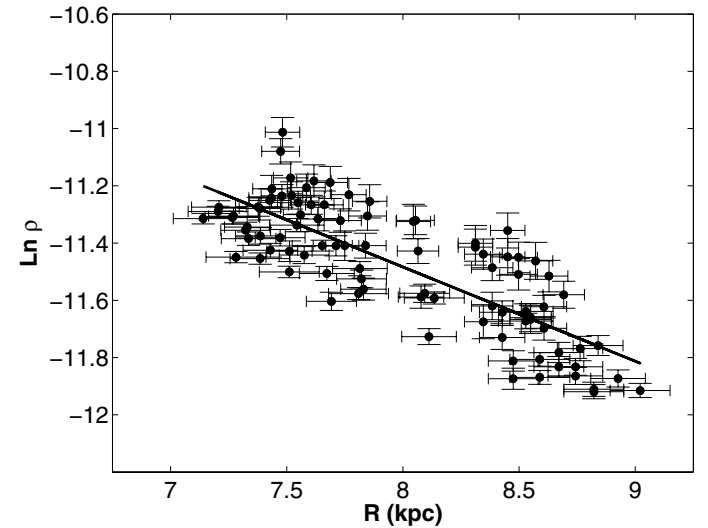

Fig. 17. Radial variation of the density for RC stars at distances $z>$ $1 \mathrm{kpc}$ and the best fit obtained. Error bars come from the Poisson noise in the counts and the uncertainty on the galactocentric distance, $R$.

Table 3. Radial parameters for the thick disc.

\begin{tabular}{ccc}
\hline \hline Range of $z$ & $\begin{array}{c}\rho_{\odot, \mathrm{THICK}} \\
{\left[\mathrm{stars} \mathrm{pc}^{-3}\right]}\end{array}$ & $\begin{array}{c}h_{R, \mathrm{THICK}} \\
{[\mathrm{kpc}]}\end{array}$ \\
\hline$z>1 \mathrm{kpc}$ & $(1.43 \pm 0.68) \times 10^{-5}$ & $3.04 \pm 0.11$ \\
$z>1.2 \mathrm{kpc}$ & $(1.38 \pm 0.67) \times 10^{-5}$ & $3.01 \pm 0.16$ \\
$z>1.5 \mathrm{kpc}$ & $(1.31 \pm 0.75) \times 10^{-5}$ & $3.02 \pm 0.05$ \\
$z>1.7 \mathrm{kpc}$ & $(1.31 \pm 0.95) \times 10^{-5}$ & $2.99 \pm 0.09$ \\
\hline
\end{tabular}

by using data corresponding to positive or negative latitudes, so we can consider this result as a good estimation of the scalelength of the thick disc.

\section{Conclusions}

With the use of a semiempirical method of extracting the stellar density from the 2MASS NIR CMDs, we have shown that the vertical distribution of the density of RC stars is better reproduced by a double exponential fit than with only the contribution of a single component. This double trend in the observed vertical distribution of the density of RC stars cannot be explained by either contamination in the counts by other populations or an effect of bias in the galactocentric distance range for the extracted stars. The scaleheights obtained in that way agree with the expected values for the sum of the thin and the thick disc of the Galaxy, thus providing a different way to analyze the presence of this component in the Galaxy. The analytical expressions obtained for the density of RC stars in the thick and thin disc are:

$\rho_{\mathrm{RC}, \mathrm{THICK}}(R, z)=1.43 \times 10^{-5} \mathrm{e}^{-\frac{\left(R-R_{\odot}\right)}{3.04 \mathrm{kpc}}} \mathrm{e}^{-\frac{|z|}{1061.9 \mathrm{pc}}} \operatorname{stars~pc}^{-3}$

$\rho_{\text {RC,THIN }}(R, z)=1.66 \times 10^{-4} \mathrm{e}^{-\frac{\left(R-R_{\odot}\right)}{2.1 \mathrm{kpc}}} \mathrm{e}^{-\frac{|k|}{268.81 \mathrm{pc}}} \operatorname{stars}^{\mathrm{pc}^{-3}}$

where the scalelength of the thin disc considered is the one given in L02. The present analysis has been restricted to the $\mathrm{RC}$ population, so the results could be different for other stars types. Also, the contribution of the Galactic halo in the counts has been neglected, as this component is dominant only at larger distances away the Galactic plane than the maximum distance considered for the RC population in this paper $(z \approx$ $2 \mathrm{kpc}$ ). Those aspects of the analysis will be addressed in a future paper.

Acknowledgements. This publication makes use of data products from 2MASS (which is a joint project of the Univ. of Massachusetts and the Infrared Processing and Analysis Center (IPAC), funded by the NASA and the NSF) and the MSX Point Source Catalog (which was obtained from the NASA/IPAC Infrared Science Archive at Pasadena, California). Thanks are also given to the anonymous referee, for helpful comments and suggestions that improved the final presentation of this paper.

\section{References}

Alves, D. R. 2000, ApJ, 539, 732

Armandroff, T. E. 1989, AJ, 97, 375

Bahcall, J. N., \& Soneira, R. M. 1984, ApJS, 55, 67

Bergeat, J., Knapik, A., \& Rutily, B. 2002, A\&A, 385, 94

Binney, J., \& Merrifield, M. 1998, Galactic Astronomy (Princeton University Press)

Buser, R., Rong, J., \& Karaaali, S. 1999, A\&A, 348, 98

Carney, B. W., Latham, D. W., \& Laird, J. B. 1989, AJ, 97, 423

Casertano, R., Ratnatunga, K., \& Bahcall, J. N. 1990, ApJ, 357, 435

Cohen, M., Hammersley, P. L., \& Egan, M. P. 2000, AJ, 120, 3362

Drimmel, R., Cabrera-Lavers, A., \& López-Corredoira, M. 2003, A\&A, 409, 205

Drimmel, R., \& Spergel, D. N. 2001, ApJ, 556, 181

Friel, E. D. 1987, AJ, 93, 1388

Garzón, F., Hammersley, P. L., Mahoney, T., et al. 1993, MNRAS, 264, 773

Gilmore, G. F., \& Reid, I. N. 1983, MNRAS, 202, 1025

Gilmore, G. F., \& Wyse, R. F. G. 1985, AJ, 90, 2015

Gilmore, G. F., Wyse, R. F. G., \& Kuijken, K. 1989, ARA\&A, 27, 555

Gilmore, G. F., Wyse, R. F. G., \& Jones, J. B. 1995, AJ, 109, 1095

Grocholski, A. J., \& Sarajedini, A. 2002, AJ, 123, 1603

Hammersley, P. L., Garzón, F., Mahoney, T., \& Calbet, X. 1994, MNRAS, 269, 753

Hammersley, P. L., Garzón, F., Mahoney, T., \& Calbet, X. 1995, MNRAS, 273, 206

Hammersley, P. L., Garzón, F., Mahoney, T., López-Corredoira, M., \& Torres, M. A. P. 2000, MNRAS, 317, L45

Haywood, M., Robin, A. C., \& Crézé, M. 1997, A\&A, 320, 440

Hoeg, E., Bassgen, G., Bastian, U., et al. 1997, A\&A, 323, L57

Larsen, J. A., \& Humphreys, R. M. 2003, AJ, 125, 1958

Liou, W. M., \& Chaboyer, B. 2000, ApJ, 544, 818

López-Corredoira, M., Hammersley, P. L., Garzón, F., Simonneau, E., \& Mahoney, T. J. 2000, MNRAS, 313, 392

López-Corredoira, M., Cabrera-Lavers, A., Garzón, F., \& Hammersley, P. L. 2002, A\&A, 394, 883 (L02)

López-Corredoira, M., Cabrera-Lavers, A., Gerhard, O., \& Garzón, F. 2004, 421, 953 (L04)

Malmquist, G. 1920, Medd. Lunds Astron. Obs., Ser. 2, 22

Norris, J., \& Green, E. M. 1989, ApJ, 337, 272

Norris, J., \& Ryan, S. G. 1991, ApJ, 380, 403

Ng, Y. K., Bertelli, G., Chiosi, C., \& Bressan, A. 1997, A\&A, 324, 65

Ojha, D. K. 2001, MNRAS, 322, 426

Ojha, D. K., Bienaymé, O., Mohan, V., \& Robin, A. C. 1999, A\&A, 351,945

Ojha, D. K., Bienaymé, O., Robin, A. C., \& Mohan, V. 1994a, A\&A, 284,810

Ojha, D. K., Bienaymé, O., Robin, A. C., \& Mohan, V. 1994b, A\&A, 290,771 
Ojha, D. K., Bienaymé, O., Robin, A. C., Crézé, M., \& Mohan, V. 1996, A\&A, 311, 456

Percival, S. M., \& Salaris, M. 2003, MNRAS, 343, 539

Paczynski, B., \& Stanek, K. Z. 1998, ApJ, 494, L219

Picaud, S., Cabrera-Lavers, A., \& Garzón, F. 2003, A\&A, 408, 141

Pietrzyński, G., \& Gieren, W. 2002, AJ, 124, 2633

Pietrzyński, G., Gieren, W., \& Udalski, A. 2003, AJ, 125, 2494

Reid, N., \& Majewski, S. R. 1993, ApJ, 409, 635

Rieke, G. H., \& Lebovsky, M. J. 1985, ApJ, 288, 618

Robin, A. C., \& Crézé, M. 1986, A\&AS, 64, 53

Robin, A. C., Haywood, H., Crézé, M., Ojha, D. K., \& Bienaymé, O. 1996, A\&A, 305, 125

Rong, J., Buser, R., \& Karaali, S. 2001, A\&A, 365, 431

Ruphy, S., Robin, A. C., Epchtein, N., et al. 1996, A\&A, 313, L21

Sandage, A. 1987, AJ, 93, 610

Spagna, A., Lattanzi, M. G., Lasker, B. M., et al. 1996, A\&A, 313, L21
Salaris, M., \& Girardi, L. 2002, MNRAS, 337, 332

Sarajedini, A., Lee, Y. W., \& Lee, D. H. 1995, ApJ, 450, 712

Sarajedini, A., Grocholski, A. J., Levine, J., \& Lada, E. 2002, AJ, 124, 2625

Siegel, M. H., Majewski, S. R., Reid, I. N., \& Thompson, I. B. 2002, ApJ, 578, 151

Skrutskie, M. F., Schneider, S. E., Stiening, R., et al. 1997, in The Impact of Large Scale Near-IR Sky Surveys, ed. F. Garzón F., N. Epchtein N., A. Omont, B. Burton, \& P. Persi (Dordrecht: Kluwer), 25

Stanek, K. Z., Udalski, A., Szymanski, M., et al. 1997, ApJ, 477, 163 Trefzger, Ch. F., Pel, J. W., \& Gabi, S. 1995, A\&A, 304, 381

Vallenari, A., Bertelli, G., \& Schmidtobreick, L. 2000, A\&A, 361, 73 Wainscoat, R. J., Cohen, M., Volk, K., Walzer, H. J., \& Schwartz, D. E. 1992, ApJS, 83, 111

Yamagata, T., \& Yoshii, Y. 1992, AJ, 103, 117

Yoss, K. M., Neese, C. L., \& Hartkopf, W. I. 1987, AJ, 94, 1600 